\title{
Outils notionnels pour l'analyse des controverses
}

Notional Tools for Controversy Analysis

\section{Agata Jackiewicz}

\section{OpenEdition}

\section{Journals}

Édition électronique

URL : https://journals.openedition.org/questionsdecommunication/11084

DOI : 10.4000/questionsdecommunication. 11084

ISSN : 2259-8901

\section{Éditeur}

Presses universitaires de Lorraine

\section{Édition imprimée}

Date de publication : 1 septembre 2017

Pagination : 137-159

ISBN : 9782814303256

ISSN : 1633-5961

\section{Référence électronique}

Agata Jackiewicz, "Outils notionnels pour l'analyse des controverses », Questions de communication [En ligne], 31 | 2017, mis en ligne le 01 septembre 2019, consulté le 26 octobre 2021. URL : http:// journals.openedition.org/questionsdecommunication/11084; DOI : https://doi.org/10.4000/ questionsdecommunication. 11084 


\title{
OUTILS NOTIONNELS POUR L'ANALYSE DES CONTROVERSES
}

\begin{abstract}
Résumé. - L'article présente une grille notionnelle destinée à guider l'interprétation des traces langagières des controverses telles qu'elles se manifestent sur la plateforme Twitter. L'idée défendue est que le profilage notionnel des productions langagières collectées, souvent foisonnantes, riches et bruitées, permet d'ordonner analytiquement le matériau discursif associé à une controverse. L'enjeu est de pouvoir appréhender des situations polémiques dans leur complexité inhérente, en tenant compte de la diversité des cibles et des points d'achoppement, des postures et des motivations des protagonistes, ainsi que des modes de validation des positionnements défendus.
\end{abstract}

Mots clés. - controverses, polémiques, grille d'analyse, marqueurs et procédés linguistiques, Twitter, mots-dièse, gestation pour autrui, procréation médicalement assistée 
L

es réseaux socionumériques constituent une arène privilégiée d'échanges sur les grandes questions ou controverses d'intérêt public, qu'elles soient d'ordre politique, sociétal, religieux ou économique. Collecter et analyser les traces verbales de ces controverses est un enjeu et une opportunité scientifique non seulement pour les sciences sociales et politiques (traçabilité du social) et l'analyse du discours (hétorique de la controverse), mais aussi et de plus en plus pour le traitement automatique des langues - TAL - et les linguistiques de corpus (veille et fouille d'opinions...). Une telle finalité nécessite des apports convergents, permettant de tenir compte des caractéristiques spécifiques aux univers discursifs à support numérique, des propriétés linguistiques des discours à caractère polémique, sans oublier la dimension sociétale des processus conflictuels qui engendrent de tels discours. Un important travail de systématisation, de modélisation et de construction de ressources associées (linguistiques et informatiques) reste encore à accomplir dans cette perspective, en établissant des ponts entre les disciplines concernées.

Les nouveaux dispositifs de communication numériques offrent des espaces d'expression et d'échange massivement investis par la société. Ces espaces médiatiques en ligne articulent différents rapports et diverses formes d'interaction, de lindividu à ses cercles sociaux, du privé au public, de l'objet à ses référencements, reprises et commentaires. Ils induisent de nouvelles formes langagières et de nouveaux genres de plus en plus complexes. Chaque production écrite ou orale (tweet, message sur Facebook, billet de blog, article de presse en ligne, vidéo...) est potentiellement commentée ou évaluée, citée ou référencée, reprise en létat, enrichie ou détournée. La collecte, la description et l'analyse de telles productions verbales dépassent largement les cadres des analyses linguistiques classiques et représentent un défi méthodologique et scientifique. Étant donnée la nature à la fois sociale, technologique et linguistique de ces objets et les problèmes d'explicitation et de formalisation que leur analyse implique, les chercheurs sont amenés à privilégier des cadres pluridisciplinaires et à construire des outillages descriptifs diversifiés.

Par son originalité disciplinaire (à l'interface de la linguistique, du TAL, des sciences de l'information et de la communication et de la sociologie), l'étude proposée se situe dans une dynamique récente qui investit scientifiquement le terrain du numérique. L'objectif général vise à construire un ensemble d'outils d'analyse et de ressources permettant de rendre compte des multiples formes d'expression et d'interaction verbales manifestant des procédures de négociation des points de vue antagonistes (Jackiewicz, 20 I6b). II s'agit de pouvoir traiter efficacement des questions complexes en évitant une approche binaire. II ne s'agit pas ici de fournir des analyses de fond d'une controverse particulière, comme c'est le cas de certains auteurs cités plus loin, mais de proposer des outils linguistiques appropriés qui permettraient d'assister un tel travail. Nous défendons l'idée selon laquelle la connaissance des mécanismes inhérents aux processus polémiques et des formes d'expression consacrées (elles-mêmes dépendantes du contexte et du dispositif de communication) permettront d'accéder de manière ciblée à des contenus qui en dévoilent des éléments d'intelligibilité. 
Le choix du réseau de microblogage Twitter comme terrain d'observation s'est imposé non seulement en raison de sa popularité comme arène de débats actuels, mais aussi en raison du format des tweets qui favorise une expression directe et synthétique des opinions. Nous voyons dans les énoncés polémiques de Twitter des modèles élémentaires de verbalisations qui peuvent composer des discours polémiques plus élaborés. Nous cherchons à y déceler les traces des fonctionnements sociaux propres aux situations de controverse. Ce travail dévoile également comment les utilisateurs de Twitter s'approprient et mettent à profit les fonctionnalités de ce dispositif en situation de polémique. Le corpus d'étude est constitué de deux collections de tweets en rapport avec un ensemble de sujets polémiques touchant au mariage, à la procréation et à la filiation. Ces questions sont de nature à trouver un très large écho dans la société civile et donc sur l'ensemble des dispositifs de communication qu'elle emploie.

L'article dresse d'abord un panorama des travaux de recherche en sciences humaines et sociales pertinents pour notre problématique. Ensuite, après avoir rappelé certaines caractéristiques saillantes du dispositif de Twitter, nous présentons le corpus d'étude et esquissons la méthode employée. L'essentiel de l'article porte sur la description de la grille notionnelle conçue pour profiler les corpus de discours polémiques et guider leurs analyses outillées.

\section{Contexte scientifique et sociétal}

\section{Bref état des lieux}

Comprendre comment se construit l'espace d'intelligibilité autour d'une controverse (avec ses points de désaccord, ses acteurs, ses jeux d'arguments et de preuves), comment se distribuent les forces et intérêts de ses acteurs, quelles sont les formes de légitimité reconnues par les protagonistes ou quels sont les modes de délibération et d'action choisis, constitue un vaste champ de recherche alimenté depuis de nombreuses décennies par plusieurs disciplines.

L'étude des controverses et, plus globalement, des processus conflictuels, a suscité une abondante production scientifique en sociologie et en science politique. L'idée générale qui motive ces travaux est de pouvoir entrer dans l'analyse du monde social par ses moments critiques en privilégiant la question des processus de dispute. Le conflit est considéré comme un élément central des ensembles sociaux, de la famille, de l'État, qui vient en opposition avec l'idée d'une unité (ou intégration) sociale et politique parfaite. C'est aussi un catalyseur social provoquant ou précipitant des évolutions. Sans exhaustivité, nous pouvons citer les contributions de Bruno Latour (2007), Cyril Lemieux (2007), ChristopheTraini (2009), Francis Chateauraynaud (20 I I) et Dominique Reynié (20I2). L'ouvrage de Francis Chateauraynaud (20I I) propose « un essai de balistique sociologique » articulé avec une sociologie argumentative 
pour rendre compte des processus dynamiques par lesquels se forment et évoluent les jeux d'acteurs et d'arguments. Nous nous réfèrerons à ce travail à plusieurs moments de notre réflexion. Des apports notables viennent des recherches visant à cartographier des controverses scientifiques perçues comme plus éloignées du citoyen lambda (n'ayant pas toujours les compétences requises pour comprendre le sujet). Pour Bruno Latour (2007), l'enjeu de la cartographie des controverses scientifiques et techniques est de réussir à « déployer les versions concurrentes des mêmes affaires scientifiques et techniques », de « retrouver une objectivité qui ne repose plus sur un silence admiratif, mais sur la gamme des avis contradictoires portant sur les versions opposées des mêmes enjeux $\rangle^{\prime}$. Classiquement, les cartographies des controverses visent une représentation synthétique des acteurs impliqués et des thèmes traités.

Au confluent de la sociologie et des sciences de l'information et de la communication, des travaux récents ont porté spécifiquement sur des questions polémiques d'ordre sociétal. À partir de l'étude des pétitions électroniques demandant l'arrêt du débat sur l'identité nationale (ou la suppression du ministère éponyme à son origine), Robert Boure et Franck Bousquet (20I I) interrogent les façons contemporaines d'intervenir dans l'espace public et de mettre en visibilité une cause. Placées dans le cadre d'une socio-sémiotique, une série de recherches tente d'opérer une articulation entre genre, médias et espace public. Observant Twitter, Maxime Cervulle et Fred Pailler (2014) décrivent finement les lignes de forces et l'économie affective des débats sur l'ouverture du mariage aux couples de personnes de même sexe.Virginie Julliard et Maxime Cervulle (20।3) étudient la construction discursive de la « différence des sexes» telle qu'elle émerge des deux controverses sociétales portant respectivement sur la parité et « le mariage pour tous ». Sujette à d'importantes tensions épistémiques et discursives, revenant cycliquement dans des débats publics (opposant les différentialistes et les universalistes), « la différence des sexes » demeure une catégorie de pensée qui structure l'espace public. Explicite ou implicite, cette catégorie est présente dans les argumentaires nourrissant la polémique sur la gestation pour autrui (GPA), comme on le verra dans la suite de l'article.

La question des propriétés caractéristiques et des modalités verbales de la délibération polémique a débouché sur de nombreux travaux en analyse du discours. Signalons d'abord les apports majeurs de Catherine Kerbrat-Orecchioni (1980, 1992, 2005), Marc Angenot (1982, 2008), Dominique Maingueneau (1983, 2008), ainsi que les recherches de Ruth Amossy et de ses collaborateurs (Amossy, Burger, $201 \mathrm{I}$; Koren, $201 \mathrm{I}$; Amossy, 2014). Des études plus ciblées, portant sur certains aspects des échanges éristiques, comme la violence verbale (insulte...), la rationalité et l'irrationalité ou l'implication émotionnelle, sont également éclairantes (Plantin, 1997, 201 I ; Desmons, Paveau 2008 ; Moïse et al., 2008 ; Lagorgette 2012 ; Ogier, 2012 ; Rosier, $2012 \ldots$.. La discussion de la spécificité du débat polémique par

I Pour un exemple de cartographie, on peut consulter : http://controverses.sciences-po.fr/archive/ biocarburants/controverse_carto.html. Consulté le 02/06/2017. 
rapport au débat argumentatif en général, entreprise notamment par Christophe Plantin (2003), constitue une autre technique pour faire ressortir ses traits les plus saillants. Sans aboutirà un consensus, les auteurs (ceux cités plus haut, mais également Dascal, 1998 ; Dascal, Chang, 2007 ; Rennes, 2007 ; et bien d'autres) proposent différentes configurations de traits, nombreux et relatifs à des réalités variées, tels polarisation et dichotomisation des thèses antagonistes, présence d'un proposant, d'un opposant et d'un tiers, violence verbale, recours à des paralogismes... On en retiendra que la notion de polémique renvoie à un phénomène sociolangagier multidimensionnel, dont les propriétés définitoires relèvent de plusieurs ordres (social, politique, psychologique, linguistique, discursif...).

L'étude des pratiques discursives propres aux réseaux et aux médias socionumériques se trouve aujourd'hui au cœur des préoccupations de nombreux chercheurs. Pour une description plus approfondie des caractéristiques des discours de Twitter, le lecteur pourra consulter (Boyd, Golder, Lotan, 2010 ; Marwick, Boyd, 2010 ;Zappavigna, 201 I ; Longhi, 2013 ; Jackiewicz,Vidak, 2014 ; Paveau, 20I5...). On trouvera une revue plus générale des problématiques et travaux concernant des réseaux socionumériques avec Thomas Stenger et Alexandre Coutant (20 I ). L'ouvrage édité par Christine Barats (2013) offre un panorama des recherches récentes sur l'observation et l'analyse du web en sciences humaines et sociales.

\section{Caractéristiques du dispositif, nature et propriétés du corpus}

Twitter constitue une sorte d'agora numérique où peuvent s'exprimer et parfois débattre des personnes qui, autrement, n'auraient pas la possibilité de se faire entendre ${ }^{2}$. Si les grands médias sont accessibles en priorité à certaines catégories d'acteurs, le web 2.0 offre des espaces d'expression à des groupes subalternes, des minorités... ce qui participe à l'émergence de visions et de définitions du monde alternatives. Tandis que les médias classiques, lesquels orchestrent en quelque sorte la diffusion des polémiques et en assument la responsabilité, les réseaux socionumériques échappent à un tel contrôle. Et l'on ne peut prétendre à une quelconque maitrise intellectuelle, technique ou stratégique ${ }^{3}$ des débats qui y prennent place. Cette accessibilité des médias sociaux modifie en retour le statut et les modalités de la prise de parole publique, tout en posant des questions liées à la démocratie numérique, à la gouvernance et à la surveillance des réseaux.

\footnotetext{
2 Dans le cadre de Twitter, il est possible de prendre part à une polémique publique, sans y être expressément invité ou autorisé. Le critère évoqué par C. Kerbrat-Orecchioni ( $1980: 23$ ) (« tout le monde ne peut à son gré pénétrer dans l'arène polémique, et tous les participants n'y luttent pas à armes égales ») n'est donc que partiellement vrai ici.

${ }^{3}$ L'investissement de Twitter par le collectif Manif pour tous, les jours des manifestations nationales, montre une conscience aigüe de l'importance stratégique d'investir et d'occuper ce réseau social pour faire entendre sa voix, mais il n'en découle pas pour autant un contrôle stratégique effectif.
} 
En effet,le dispositif communicationnel et énonciatif deTwitter produit des conditions spécifiques d'échange polémique, différents de ceux qui sont habituellement analysés (Amossy, 2014) - débats ou duels télévisés, séries de discours monogérés compilés et rapportés par des médias. Dans la sphère du web, les échanges polémiques sur des forums de discussion ou des pages de commentaires associés à des articles se rapprochent des tweets par leur aspect polylogue ${ }^{4}$ et le caractère anonyme des participants, mais s'en éloignent par plusieurs points : absence d'ancrage, absence de modération, ampleur de la participation, forme courte, pratiques d'adressage et de reprise...

Un autre point semble important à souligner. Si Twitter peut être vu comme un lieu de confrontation des opinions, le mode d'expression et d'interaction induit par son dispositif favorise en réalité une multiplicité de micro-débats. Souvent, les opinions évoluent en parallèle plutôt qu'elles ne se confrontent véritablement.

\section{Choix des observables}

Le corpus d'étude comprend deux ensembles de tweets collectés automatiquement, concernant respectivement: (i) l'ouverture du mariage aux couples de personnes de même sexe (corpus 《 MAR ») ; (ii) la filiation issue de la GPA (corpus « FIL »). Les tweets sont datés pour permettre d'adosser l'analyse aux événements qui ont constitué les temps forts de ces deux controverses (débats, auditions à l'Assemblé nationale, manifestations nationales...). Le corpus FIL, notre corpus principal, a été collecté le 5 octobre 2014, durant 24 heures, à partir des mots clés suivants : pma, gpa, procréation pour autrui, gestation pour autrui, filiation, famille, manif pour tous, manifpourtous. I| comprend au total 184300 tweets. En simplifiant, la mobilisation organisée ce jour servait à montrer l'opposition à la GPA et à installer dans l'espace public la cause d'une filiation « naturelle », vécue comme menacée par le récent accès au mariage accordé aux couples homosexuels.

Contrairement à des controverses scientifiques ou théologiques par exemple, la spécificité de cette controverse est qu'elle n'est pas l'apanage d'une catégorie d'experts ou de spécialistes, mais concerne la société entière, même si elle ne touche directement que certaines catégories de ses membres. C'est la question de la filiation pour les enfants nés de la GPA et de la PMA qui se trouve au cœur des discussions. Le généticien Axel Kahn (2010 : 146-147) explique ainsi ces deux pratiques :

« La gestation pour autrui, c'est la pratique de la mère porteuse, technique qui permet de porter un enfant en dehors de tout rapport charnel, à partir des ovocytes d'une autre femme. La procréation pour autrui se distingue de la gestation pour autrui. Dans le premier cas, la femme qui porte l'enfant est sa mère génétique : dans le second cas, elle n'est que sa gestatrice, l'enfant ayant été conçu avec les gamètes du couple demandeur ou du tiers donneur. Cette technique d'insémination artificielle

\footnotetext{
${ }^{4}$ Le terme polylogue qualifie un dialogue engageant de nombreux locuteurs effectifs ou potentiels, comme dans un forum de discussion sur le web.
} 
remet en cause une règle fondamentale du droit de filiation. En droit français, la mère est la femme qui accouche "mater semper certa est" (plutôt "mater certissima, pater semper incertus" ?). Si le père n'est pas certain, la mère est ».

La controverse qui en découle est résumée comme suit:

« Comme dans la majorité des situations, la controverse éthique porte sur l'intérêt de l'enfant à naître, le risque d'exploitation des femmes, la marchandisation des corps, l'atteinte à leur dignité. La gestation pour autrui est une situation qui implique la présence de trois, quatre, voire cinq acteurs. II y a le couple, la mère porteuse avec laquelle on passe un contrat, un médecin, éventuellement une donneuse d'ovule, etc. La mère porteuse prend en principe l'engagement d'abandonner l'enfant à la naissance, ce qui n'est pas rien. Cela signifie qu'elle s'engage par contrat à n'être en la matière qu'une matrice, en aucun cas une mère. En d'autres termes, de tels contrats équivalent à des locations temporaires, ou au moins d'aliénation d'utérus, assortis de l'offre en général payante d'un service gestationnel » (ibid. : 147).

\section{Caractéristiques du corpus, caractéristiques des tweets}

Pour revenir à notre corpus d'observation, plusieurs remarques préliminaires s'imposent. Les contenus extraits de Twitter ne représentent pas des ensembles clos, complets et cohérents de significations, et cela quel que soit le mode de collecte déployé. C'est une portion d'un vaste interdiscours qui renvoie à un espace écologique complexe (les tweets indexés par les mêmes mots-dièse, les retweets, les contenus connexes accessibles via des liens hypertextes...). Cet espace fait à son tour partie d'une configuration plus vaste qui associe étroitement les productions de la presse imprimée et en ligne, celles de la radio, de la télévision... Chaque tweet est porteur d'un message court qui représente une information, une opinion, un argument. . . II rencontre ou croise des milliers d'autres propos, indexés pour certains par des mots-dièse et des mentions. On a donc affaire à une constellation verbale, $a$ priori impossible à délimiter, car prise dans une dynamique mouvante et incessante des flux médiatiques et des interactions. C'est une narration fragmentée du monde, faite de manques et d'incohérences, mais actualisée en permanence. Néanmoins, on peut espérer pouvoir y trouver une forme de pensée collective, développée à plusieurs voix, laquelle mérite, à nos yeux, d'être considérée et interrogée.

À l'évidence, ce corpus ne comporte pas uniquement des traces des débats entre acteurs directement engagés dans la controverse, mais, plus largement, des messages de toute personne qui souhaite s'exprimer à ce sujet. La distinction classique entre les acteurs de la controverse et le tiers écoutant semble ici être mise à mal. Les modes d'action et les motivations des participants peuvent être très divers. II y a ceux qui prennent position, ceux qui informent ou relaient l'information, ceux qui posent des questions, ceux qui témoignent, ceux qui appellent à l'action ou qui « combattent », ceux qui dévoilent leurs sentiments (indignation, colère, exaspération...), ceux qui emploient la dérision, ceux qui cèdent au plaisir du bon mot (« Envisager la GPA, c'est faire des plans sur la gamète », 05/ | 0/20 |4). Et ce n'est pas tout. Car, nous ne 
pouvons faire abstraction ici des pratiques malveillantes de flaming ${ }^{5}$ et de trolling. L'envoi de ces éléments, sciemment perturbateurs et délibérément polémiques, a pour conséquence de baisser le rapport « signal-bruit ». En pratique, ce type de tweets n'est pas aisé à identifier, sauf s'il s'agit d'un acte revendiqué.

Cette diversité des attitudes se reflète logiquement dans le format et le contenu des tweets. Certains relèvent clairement d'un modèle agonistique (défendre ses positions de façon argumentée, critiquer celles de son adversaire), d'autres favorisent un modèle oratoire fondé sur l'expression des émotions et des passions, d'autres encore conjuguent ces deux modes. Tout tweet de cette collection ne comporte pas obligatoirement de marque de discordance. Certains, de nature plus didactique que polémique, se présentent sous une forme parfaitement neutre. Comme dans toute situation de communication, l'humour, l'ironie, le doute ou une distance affichée peuvent témoigner d'une certaine ambivalence face au débat ou à son objet. II n'est pas rare que le contexte extralinguistique joue sur l'interprétation polémique des tweets en apparence neutres, dont certains mots fonctionnent comme des rappels mémoriels à des événements socialement marquants (dans « Une famille c'est un papa, une maman, des enfants et un congélateur », le mot congélateur renvoie aux affaires des bébés congelés ${ }^{7}$ ). Enfin, autant le dire ouvertement, la signification d'un tweet isolé est fragile. Dans nos collections, nombreux sont les tweets dont nous ne pouvons interpréter la teneur. Coquilles ou fautes d'orthographe, maladresse rédactionnelle, contradictions, implicite, intentions floues... sont autant d'obstacles.

\section{Démarche et méthode}

Pour travailler sur de tels corpus, on est amené à construire une démarche adaptée. La nôtre peut être résumée comme suit. Dans un premier temps, à partir des travaux de référence en sociologie, science politique et analyse de discours et en appui sur le corpus FlL, nous avons élaboré une grille d'analyse permettant de profiler le corpus et de l'interroger selon différentes facettes de la conflictualité (plans, protagonistes, logiques... ). Dans un deuxième temps, chaque notion du modèle a été caractérisée par un champ lexical caractéristique qui forme sa signature lexicale. La projection des lexiques sur le corpus a permis de

\footnotetext{
${ }^{5}$ Le terme flaming désigne une pratique consistant à poster des messages délibérément hostiles, insultants et généralement avec l'intention de créer un conflit sur un groupe de discussion (sur Usenet), un forum (sur un site web) ou une liste de diffusion (par courrier électronique).

${ }^{6}$ Le terme trolling désigne une pratique visant à créer artificiellement une controverse qui focalise l'attention aux dépens des échanges et de l'équilibre habituel de la communauté. En argot internet, un troll caractérise ce qui vise à générer des polémiques : un message (par exemple sur un forum), un débat conflictuel dans son ensemble ou la personne qui en est à l'origine. Voici un exemple de troll trouvé dans le corpus : « L'État islamiste soutient la \#ManifPourTous 》 (05// 0/20 I4).

7 || s'agit de plusieurs cas d'infanticide multiple de nouveaux nés, dont les corps ont été dissimulés dans un congélateur.
} 
sélectionner les ensembles de tweets correspondant à chacune des dimensions de la controverse (corpus profilé). À partir de ces données, nous avons pu apprécier la présence dans le corpus et la rentabilité des lexiques définis a priori. Cette opération a permis de s'assurer que l'ensemble des dimensions du modèle sont représentées dans le corpus d'observation, ce qui confirme que le corpus renferme bien des productions verbales émises en situation de controverse. Nous avons réitéré ce traitement sur le corpus MAR, avec des résultats sensiblement comparables.

L'analyse linguistique des tweets ainsi catégorisés a conduit à l'identification des schémas d'expression caractéristiques et de certains procédés discursifs (argumentatifs, énonciatifs, rhétoriques), impliqués dans le marquage des énoncés de nature polémique. Dans un troisième temps, nous avons étendu et systématisé le répertoire des procédés linguistiques pertinents par un va-et-vient entre le corpus et des études linguistiques spécifiques portant sur des phénomènes identifiés dans le corpus (négation polémique, reformulation polémique, termes d'adresse, etc. $)^{8}$. La perspective adoptée est donc celle de la sémantique discursive, que nous conjuguons avec des techniques d'analyse plus formelle (détection de n-grams, définition de patrons linguistiques...).

\section{Modélisation notionnelle}

L'objectif est de caractériser finement les différentes formes d'expression et d'interaction verbale manifestant des procédures de négociation de points de vue antagonistes ou divergents. Or, ces procédures sont naturellement complexes. L'affrontement des positions conflictuelles peut porter sur une diversité de points et les modes de confrontation verbale sont nombreux. Pour pouvoir délimiter le matériau textuel pertinent et guider son analyse, nous avons besoin d'un ensemble de notions qui définissent et structurent une situation de controverse.

Partant d'une sélection de travaux de référence en sociologie des conflits, science politique et analyse de discours, nous avons élaboré une grille d'analyse qui renvoie à différentes facettes de la conflictualité. II s'agit d'un ensemble de notions qui ont trait à la nature des entités ciblées dans le débat, à l'identité, aux postures et aux motivations de ses protagonistes, ainsi qu'aux modes de validation critique des positionnements adoptés. Sans viser une hypothétique complétude ou une forme de vérité ultime sur la réalité d'une controverse, l'idée est de faciliter la compréhension des processus éristiques dans leur complexité, grâce au profilage notionnel des énoncés et à leur analyse linguistique. Par ce moyen, on cherche à ordonner analytiquement le matériau discursif associé à une controverse.

\footnotetext{
${ }^{8}$ Par manque de place, nous n'indiquerons ici que les procédés les plus caractéristiques.
} 


\section{Plans fondamentaux}

En suivant Francis Chateauraynaud (2011 : 104), nous considérerons que les argumentateurs engagés dans une controverse se trouvent aux prises avec des questions relevant de trois plans différents : ontologique, épistémique et axiologique. Chacun de ces univers offre une fenêtre spécifique sur la controverse, ses objets et ses acteurs.

\section{Plan ontologique}

Le plan ontologique renvoie aux entités pertinentes du débat et à leurs modes d'existence, qui font ou ne font pas l'accord. On peut y situer également des questions d'ordre terminologique, car s'entendre sur les objets implique de s'accorder sur les mots choisis pour les désigner, tant dans leur intension que dans leur extension. Une sorte de « lutte pour la signification » peut s'installer quand les représentations associées aux objets du débat divergent trop fortement.

La perspective ontologique, focalisée sur les objets du débat, permet de connaître, notamment à travers des énoncés de type définitoire ( $X$ est un $Y$ ) ou de négation polémique ( $X$ n'est pas un $Y$ ), la perception qu'en ont les acteurs de la polémique. Les différentes façons de désigner une même réalité ne sont pas équivalentes argumentativement (phénomène de nomination argumentative). En effet, toute définition est une interprétation et une simplification. Toute catégorisation construit de l'objet une représentation particulière. Celles que l'on propose dans un contexte polémique servent à orienter dans le sens de la thèse que l'on soutient. On notera que l'exercice de l'analogie et de la métaphore peuvent jouer le même rôle. Ces figures, qui s'adressent à l'imagination, sont employées comme catalyseurs de compréhension. De plus, elles influencent et orientent fortement la saisie intellectuelle des faits débattus. Sur le plan ontologique, nous sommes en général en présence d'énoncés qui relèvent de l'opinion (au sens de l'opinion épistémique). Certains sont en même temps porteurs de jugements de valeur.

Sur les réseaux sociaux, les phénomènes que nous venons d'évoquer se présentent sous une forme concise et condensée. La transformation d'un terme en mot-dièse (\#GPA, \#famille) constitue un procédé spécifique permettant de donner corps à la notion qu'il dénote et de l'imposer comme topic de discussion? :

I. «La \#GPA existe. Les femmes vont en Europe \#5oct $»^{10}$

2. «La GPA c'est la marchandeisation de l'humain. http:// »

\footnotetext{
${ }^{9}$ Ici, et par la suite, nous soulignons.

${ }^{10}$ Tous les tweets rapportés ici ont été rendus anonymes. Ils ont également été allégés de liens hypertextes.
} 
3. « \#GPA: L'humain vu comme un vulgaire \#ProduitDeConsommation? N'y a t-il pas assez d'\#Enfants \#Orphelins dans le monde à \#Adopter? \#Famille »

4. "La \#GPA n'est pas nécessairement une "marchandisation du corps", elle peut se faire gratuitement, comme au Canada. C'est n'importe quoi ! »

5. « La \#GPA en fait c'est de la chimie de bébés... \#France2 \#degeu \#|3h|5 »

6. « Obscurantisme, esclavage... Tous des termes inappropriés. Je comprends qu'on soit contre mais changez vos mots \#ManifPourTous \#France3 \#gpa »

\section{Plan épistémique}

Le plan épistémique s'intéresse aux modes de connaissance et de raisonnement. Comment se construit l'espace d'intelligibilité autour de la question débattue? Quel est le degré d'incertitude ou d'indécidabilité des savoirs disponibles? Quels sont les points d'achoppement? Quels sont l'outillage intellectuel nécessaire et les preuves disponibles ou attendues? En conséquence, le plan épistémique renvoie à l'ensemble des ressources intellectuelles (raisonnements, témoignages, démonstrations, mesures, expériences...) employées par les protagonistes pour faire valoir leurs points de vue.

Du fait des objectifs visés, les argumentations sont en général sélectives. II s'agit d'utiliser ce qui permet de justifier ou conforter une thèse. Les adversaires, d'une manière tout aussi sélective, se chargent des « oublis 》 et des insuffisances. Souvent, les règles du raisonnement font elles-mêmes l'objet du dissensus, voire de l'attaque verbale (par exemple, dénier la rationalité à son adversaire, en le traitant d'irrationnel, en disant qu'il délire...). Ce plan accueille donc des jugements d'appréciation visant la validité des connaissances et des raisonnements (qui en fournissent une sorte de caution épistémique). Dans la palette des procédés linguistiques caractéristiques, nous trouvons des modalités de prise en charge énonciative, certaines formes d'appréciation, l'expression des rapports de cause et de conséquence, des marques d'opposition, etc. :

7. «Le problème avec la loi \#Taubira, c'est le flou, les incertitudes de filiation, et la stigmatisation de ceux qui déplorent tout... »

8. « I 3h | 5 Très beau reportage sur la GPA, j'étais contre maintenant j'ai I sérieux doute et je comprend mieux »

9. «Si on suit la logique de la \# manifdelahonte : parents gay = enfants gay. Donc parents heteros = enfants hétéros. \# OhWait »

10. «"La GPAn'estrien d'autre que la commercialisation d'enfant"-"Sale haineux! Fasciste!!'Raisonnement de bulot cuit.»

II. «Les manifestants catho qui font des gosses a gogo parce que c'est la VIE, mais qui refusent PMA GPA. C'EST LOGIQUE? ? On en parle? » 


\section{Plan axiologique}

Le plan axiologique est celui des jugements moraux. II concerne les principes au nom desquels s'affrontent les protagonistes. II semblerait que, dès qu'il y ait controverse, il y ait engagement implicite ou explicite des valeurs. Le plan axiologique sert en quelque sorte de toile de fond. II renvoie aux repères moraux d'une société ou d'un groupe d'individus, faits de principes, de normes et de valeurs (Jackiewicz, 20 I 6a). Souvent donc, l'argumentateur cherchera à imposer ou à défendre son point de vue, en invoquant des valeurs, qu'il n'hésitera pas à qualifier de communes, fondamentales, fondatrices, cardinales, précieuses, vraies...

Normes et valeurs ne sont ni immuables, ni incontestables, c'est leur acceptation par le groupe qui fonde leur existence. De véritables querelles à propos des valeurs peuvent avoir lieu : pour défendre des valeurs menacées, bafouées ou affaiblies, pour s'affranchir des normes trop étroites ou contraignantes, pour réclamer la reconnaissance d'autres principes, etc. Parfois, certaines valeurs peuvent être en contradiction. De « nouvelles » valeurs peuvent venir bousculer les « anciennes 》. Se posent alors des questions de choix ou de hiérarchisation de ces valeurs. Si comme le suggèrent certains sociologues, « nous préférons réviser ce qui est pour nous le moins fondamental » (Livet, cité in : Chateauraynaud, 201 I : 97), dans une situation de controverse, il est important de comprendre selon quelles logiques ses protagonistes tracent une frontière entre ce qui est acceptable (ou révisable) et ce qui ne l'est pas.

12. « II y a un principe fondamental du droit et de la médecine qui a pour nom : l'indisponibilité du corps humain. \#GPA \#PMA ventes d'organes... »

13. « la famille est la valeur refuge à protéger en priorité »

14. « \#bourdindirect Une famille homo vit en dehors des normes de la reproduction humaine donc au sens biologique ce n'est pas une famille »

15. «Vous ridiculisiez la France avec vos principes d'un autre temps, d'une idéologie dépassée et usée. \#LaManifdelahonte \# ... »

16. «Mais fichtre, qu'est-ce donc que la \# filiation? II existe encore des gens qui croient que le lien du sang est une valeur en soi?»

17. « "La famille aujourd'hui ce n'est pas un modèle unique. Au final la valeur fondatrice de la \#Famille c'est l'\#Amour" @xx@xx \#LMPT »

\section{Les protagonistes}

Toute controverse ou polémique implique des participants ; on parle aussi d'acteurs ou de protagonistes. Celui qui argumente se trouve devant un auditoire à convaincre et en face d'un adversaire réel ou potentiel défendant une position différente ou opposée. Classiquement, on met l'accent sur l'existence d'une 
relation triadique impliquant trois actants : les deux parties adverses, le proposant et l'opposant, ainsi que le tiers qui renvoie à la catégorie des observateurs (Plantin, 2003 : 383). Cette tripartition idéale se manifeste-t-elle systématiquement dans toute forme de polémique ou de controverse ? Ou existe-t-il d'autres configurations remarquables (impliquant une hiérarchie d'acteurs"', par exemple)? L'opposition entre experts et profanes est-elle toujours pertinente? II semblerait que, dans le cas des polémiques sociétales, parler d'expertise plurielle serait plus approprié. La question d'identification des publics concernés (au sens large) par une controverse est donc au cœur des préoccupations de l'analyste. Dans cette perspective, il peut être éclairant d'observer comment les discours polémiques nomment, catégorisent et partitionnent leurs différents acteurs.

La problématique des protagonistes amène avec elle des questions identitaires, ellesmêmes liées à des logiques d'appartenance, des principes de loyauté et des liens d'obligation entre acteurs individuels et collectifs. L'identité mise en jeu est souvent fondée sur des valeurs de groupe. L'énoncé de la position défendue peut-être soustendu par l'évocation de l'appartenance catégorielle de son porteur (femme, gay, catholique...) et/ou des valeurs partagées par les membres de cette catégorie.

À la lumière du corpus FIL, on voit que plusieurs catégories d'entités sont directement concernées par la GPA : les enfants à naître, les femmes porteuses, les donneurs... Des mots-dièse sont créés pour les désigner, souvent avec une épaisseur sémantique argumentativement significative (\#enfant, \#Orphelins, \#mèresporteuse, \#interetdelenfant, \#EnfantsOtages, \#EnfantMarchandise, \#TheyDetestChildren...).

L'évocation de l'adversaire (discursif) peut aller de la désignation nominale à la simple allusion. Collectif, celui-ci peut être identifié (et épinglé) au moyen des tours tels tous ces $X$ qui, les petits $X$, les bons $X$, les groupuscules $X \ldots$... Les discours polémiques livrent des représentations stéréotypées des acteurs impliqués dans le débat. Dans le corpus FIL, on trouve les figures du gay, du socialiste, du bobo, du catholique intégriste (de droite)... désignés par des mots-dièse forgés pour l'occasion (\#lesbienne, \#gaucho, \#gauchetaree, \#gauchosphère, \#tarésocialos, \#SocialosOut, \#BadinterOut, \#CathosIntégristes...).

Enfin,il convient de souligner que le corpus flL comporte de nombreux tweets postés par des observateurs non impliqués dans la polémique. Ceux-ci marquent principalement une prise de distance vis-à-vis du sujet de la controverse et de ses protagonistes (23) :

18. «En tant que femme, il serait impensable de ne pas soutenir la combat contre la \#GPA qui nie toute forme de dignité humaine. \#FamillesEnAvant »

19. « Tous ces \#gaucho qui haïssent nos anciens et qui préfèrent la sodomie.. \#triste \#ManifPourTous »

20. «Dans la \#GPA, 3 personnes impliquées : le donneur de sperme, la donneuse d'ovocytes et la mère porteuse. \#|3h|5 »

\footnotetext{
" C. Kerbrat-Orecchioni (1980: 19) mentionne une possible présence d'un actant supplémentaire, une sorte d'allié ou de supporteur d'un des polémiqueurs principaux, qualifié d'« adjuvant ».
} 
21. «De plus en plus de \#Français favorables aux \#mèresporteuses:http://via@xx\#ManifPourTous \#GPA \#PMA. 》

22. «Aux femmes partisanes la GPA : Qu'attendez-vous pour faire des enfants que vous offrirez "généreusement" à des couples stériles? »

23. «Donc si je comprends bien, la france des bobos se déchirent sur la \#PMA ...... Mais, le peuple s'en fout. Eux veulent qu'on parle emploi »

\section{Logiques d'acteurs}

Afin de cerner plus finement les différentes logiques possibles dans lesquelles peuvent s'inscrire les acteurs de la controverse, nous proposons de distinguer trois ensembles notionnels, renvoyant respectivement aux postures, aux motivations et aux garants argumentatifs. Chacune des catégories se décline selon plusieurs cas de figure, impliquant des positionnements et interactions des protagonistes du débat (des proposants, des opposants, des observateurs, des médiateurs...).

\section{Postures}

Les attitudes et les modes d'implication des acteurs des controverses peuvent être variés. En simplifiant, on dira que certaines se situent plutôt du côté du dialogue, d'autres sont davantage tournées vers l'action. Pour marquer la différence entre ces deux types de posture, Francis Chateauraynaud (20II : 3I) parle de « disjonction théorique entre argumentation et mobilisation ». D'autres couples de termes sont employés en sociologie pour désigner la même réalité : « posture régulatrice $» /$ »posture radicale $»$, , paradigme de dialogisme $» / 《$ paradigme de conflictualité », « agir communicationnel »/《 agir stratégique ».

Une posture régulatrice se traduit par une attitude de vigilance et d'alerte, attentive aux signes précurseurs, aux prodromes ou encore aux premières manifestations d'un problème, qu'il s'agira de porter à la discussion publique. De même, toute forme de questionnement et de réflexion sur le problème posé en relève pleinement. Cette posture s'accompagne préférentiellement d'une intention persuasive, où celui qui argumente vise à amener son interlocuteur à changer d'avis, à adopter totalement ou partiellement son point de vue.

Cette logique de vigilance et de dialogue se reconnaît par un ton modéré et corrélativement par l'absence de violence verbale, l'emploi des verbes d'intellection (réfléchir, s'interroger, se demander, comprendre...) et des formes interrogatives :

24. « \#5oct Sachons dépasser l'affect qui conduit à accepter la \#GPA en amenant à réfléchir sur ce que ça veut dire, pour la mère et pour l'enfant » 
25. « Je me demande comment plus tard remonter dans l'origine d'une famille avec la GPA ?»

26. « RT @Xx@xx Merci à toi, je suis consciente que c tabou, je dis juste vigilance aux autorisations de GPA. Bonne journée... »

27. « j'suis sceptique : quels avantages de la GPA et PMA par rapport à l'adoption ? »

28. «PMA, GPA : les clés pour comprendre le débat »

29. «D'aprés sondage \# BFMTV La Majorité des Francais est pour mariage gay GPA PMA SVP faites I référendum pr régler le pb une bonne fois pr toutes »

Tournons-nous maintenant vers le deuxième type de posture, qualifié de radical. On note ici un basculement vers un durcissement irréductible des positions de locuteurs devenus adversaires. Le répertoire argumentatif cède la place au répertoire d'action, voire de combat. II s'agit de passer en force, au lieu d'essayer de convaincre.

Ce paradigme renvoie aux actions de mobilisation et d'affrontement, plus radicales, tournées vers une « victoire ». Les traces du positionnement discursif des acteurs qui sont mobilisés pour ou contre un projet (une reforme...) passent a minima par la présence de termes comme mobilisation, revendication, action de rue, slogans, mots d'ordre... Le registre de l'affrontement est signalé par des mots renvoyant explicitement aux différentes formes de combat (lutte, bataille, révolte, épreuve de force...). En font partie également des énoncés qui donnent une vision antagoniste de la réalité ou qui expriment un fort clivage entre les protagonistes (ça/ces gens...). Le désaccord contribue à clarifier l'identité respective des opposants (différentiation avec autrui, tissage du lien entre alliés...), à la création de communautés et d'échelles ou de stratifications sociales... Un engagement de groupe peut d'ailleurs conduire à une résistance accrue aux arguments de l'adversaire. On agit en tant que $X$, au nom des X... Ce qui peut conduire certains membres des groupes concernés à refuser une telle délégation, par exemple, via le mot-dièse \#notinmyname:

30. « Bravo à la \# Manifpourtous belle mobilisation équivalente à celle de février, preuve que le mouvement ne s'essouffle pas! \# onlr»

31. «Manifester pour que des gens n'accèdent pas à des droits plus que légitime, elle est belle la droite \# ManifDeLaHonte »

32. « Donc pour soit-disant protéger l'intérêt des enfants, des gens font défiler les leurs bâillonnés... \# ManifDeLaHonte »

33. « Si vous pensez que les enjeux liés à la PMA / GPA vont se régler à coup de slogans et de manifs, 'est un vrai déni de réalité ! \# ManifDeLaHonte »

34. «"Les réseaux sociaux, champs de bataille des pro et anti-Manif pour tous". »

35. «Mais les mecs de la \# ManifPourTous ils ont compris qu'ils avaient perdu le combat et que ca servait à rien de continuer à protester? » 


\section{Motivations}

La question des motivations et des raisons d'action constitue un autre paradigme des controverses. Il permet de savoir sur quels terrains se positionnent préférentiellement et s'affrontent les adversaires. Celui du progrès, celui de la justice sociale, celui de l'éthique? Ces différentes problématiques sont d'ailleurs souvent étroitement liées :

36. « La GPA est un commerce immoral. et encore plus pour des homos. Ce n'est pas cela le progrès scientifique. »

La décision de faire évoluer ou, au contraire, de maintenir des pratiques sociales bien admises jusqu'alors, mais qui apparaissent progressivement insatisfaisantes voire répréhensibles à certains, engendre toutes sortes de dilemmes moraux et suscite à chaque époque d'importantes controverses. Ce type de débat, centré sur le progrès, révèle comment s'élaborent les visions du futur. Certains adoptent une rhétorique du changement pour insister sur la nécessité d'évoluer, de s'aligner sur des solutions mises en place ailleurs ou de combler un retard. Dans le même registre, d'autres choisiront de fustiger l'immobilisme, le conservatisme ou le surinvestissement du passé. En réponse, on brandira les risques de dérive et de perte des repères. On insistera sur la défense des valeurs et des principes communs.

Différents schémas argumentatifs peuvent être déployés dans cette perspective. Sur le plan lexical, en plus des mots qui relèvent directement du registre concerné (progrès, évolution, moderne, retard, rétrograde...), on note une présence significative de termes qui renvoient à la société (société, civilisation, française...), ainsi qu'au temps (siècle, époque, temps, avenir, passé, demain, bientôt, désormais...).

37. «"La \#GPA est une évolution incontournable, je crois que si elle est maîtrisée, elle est acceptable, et j'y suis donc favorable" \#Valls 09/I I »

38. « Parce que c'est etre IN et de SON TEMPS que d'approuver le \#MPT et autre \#GPA \#PMA et qu'aujourd'hui de mettre nos valeurs au rebut?»

39. «Par contre les amalgames. C'est pas une manif " anti-gay " mais juste anti-progressiste! Faut accepter le changement de mœurs \# ManifDeLaHonte »

40. « Je vis dans une société qui trouve progressiste et moderne de se payer le ventre d'une femme. Chacun sa conception du progrès. \# GPA »

41. « La notion de famille a bcp évolué ds les faits ms les mentalités elles, sont restées figées ! »

42. « \# ManifDeLaHonte La \# France_rétrograde! Un des seuls pays modernes et démocrates à poursuivre un combat injuste et perdu d'avance. \# UMPFN »

La justice et l'égalité en droit représentent un motif souvent mis en avant dans des polémiques sociales ou politiques. Rappelons que les notions d'équité et de proportionnalité régissent grand nombre de nos jugements moraux. Par exemple, pourront être invoquées une discrimination ou l'absence d'un traitement équitable touchant une catégorie de la population. Selon la stratégie adoptée, l'argumentaire portera sur la nécessité d'assurer l'égalité de tous ou sur la défense des intérêts de 
la population flouée. On dénoncera la politique de « deux poids et deux mesures ». À ce titre, la devise républicaine « Liberté, Égalité, Fraternité » pourra être rappelée ou détournée ironiquement. Les opposants de l'égalité juridique avanceront de leur côté l'argument de l'incommensurabilité, posant comme « essentielle » et irréductible la différence entre les catégories concernées (homme/femme, couples hétérosexuels/couples homosexuels...).

Dans cette stratégie différentialiste, quand le débat touche à la structure de la famille, la parité revendiquée (y compris dans le couple) repose sur la différence des sexes posée comme naturelle et fondamentale. Cette opposition entre les positions universalistes et différentialistes s'est clairement manifestée lors des débats sur le mariage et la filiation.

Pour Juliette Rennes (2007 : 93), il existe une rhétorique commune aux demandes d'égalité en démocratie ${ }^{12}$, que ces demandes concernent les femmes, les étrangers, les homosexuels, les handicapés, etc... Dans le registre lexical, en plus des mots qui renvoient à l'équité ou à son absence (égalité, discrimination, partialité....), on notera la présence du lexique du droit et des institutions (loi, droit, constitution, gouvernement, démocratie, république...):

43. « Hé, les fascistes de la \#ManifDeLaHonte, et si vous manifestiez pour plus de justice sociale? Vous ne valez pas mieux que les tarés D'ElL »

44. « PMA : Sarkozy veut inscrire la discrimination dans la Constitution »

45. « “Liberté, Egalité, Fraternité", mais que pour les blancs hétéro catholiques c'est ça ? \#ManifDeLaHonte »

46. « Eh oui, une égalité mal comprise peut être source d'injustice. La \# GPA en est la preuve »

47. « La logique des anti-égalité de la \#ManifPourTous »

48. «La seule et la vraie \#manifpourlegalite c'est la \#manifpourtous qui défend la parité et le droit des enfants à avoir un père et une mère !. »

Les débats sociaux investissent souvent le terrain de l'éthique et de la morale. II y est question des normes et valeurs qui dictent des principes de fonctionnement des groupes et des nations, en en assurant la cohésion. L'ancrage dans une communauté de valeurs constitue d'ailleurs un facteur de construction identitaire.

Les références aux normes et valeurs, souvent présentées comme des impératifs supérieurs, universels ou universalisables, interviennent quand il s'agit de préconiser ou de refuser une décision. Normes et valeurs sont évoquées également pour la critique : celle des états de choses subis, des personnes et de leurs comportements (manquements aux devoirs, manque d'intégrité...). Normes et valeurs ne sont ni immuables, ni incontestables. Leur existence et leur acceptation sont donc souvent débattues.

\footnotetext{
${ }^{12}$ Ce disant, J. Rennes (ibid.) pose l'existence d'un type particulier de controverse qu'elle nomme « controverse d'égalité ».
} 
Les débats autour de la GPA ont réactivé la question du statut de la famille et des liens de filiation. Pour les militants de la Manif pour tous notamment, certaines valeurs ne sont pas révisables. La famille traditionnelle, au cœur du débat, est vue comme une valeur en soi, chère, refuge, cardinale, fondatrice, qui monte... Elle sert de cadre pour une filiation et une maternité naturelles. Le couple parental, 《 procréateur, asymétrique et hétérogène $»^{13}$, implique une différence des sexes, naturelle et universelle. En conséquence, les pratiques de la GPA sont qualifiées comme étant contre nature. Les voix favorables à la GPA brandissent, quant à elles, le principe d'égalité. Elles s'opposent à l'enfermement des humains dans des distinctions naturelles et à l'institutionnalisation du biologique. Des témoignages évoquant des situations familiales moins typiques ou hors norme, mais vécues comme heureuses et donc souhaitables, se font entendre:

49. «Je viens défendre la famille naturelle. Toutes les civilisations se sont effondrées dans l'homosexualité, la Grèce »

50. «Desceller entièrement la filiation de l'ordre naturel sous prétexte de son injustice signifie ouvrir grand la porte à l'eugénisme de masse.»

5I. «"La \#Maternité, précieux élément du patrimoine de l'humanité : à ne pas occulter, escamoter ni éclater" \#GPA \#50ct »

52. « \# Ludo " La \# GPA éthique n'existe pas! Respectons un principe fondamental du droit français 'Le corps est indisponible"” \# FamillesEnAvant »

53. « Ok argument invalide mais là on parle de valeur, d'un principe fondateur de la RF : l'égalité. \# Irt \# ManifPourTous »

54. « Nan! Ton modèle de la famille n'est pas le mien ... @xx J'ai été élevée que par des femmes ;-) Rassure-toi tout va bien! »

\section{Garants argumentatifs}

Pour convaincre ou réfuter les argumentations polémiques ont besoin de s'appuyer sur des éléments qui constituent de bonnes raisons d'admettre les conclusions proposées. La réalité des faits, la légitimité des débatteurs, la qualité des raisonnements font partie des principaux moyens de fonder et d'étayer les raisonnements produits.

Les faits (éléments observables directement ou pouvant être rapportés par des témoins) sont souvent exploités à titre de preuve. En particulier quand ils renvoient aux expériences personnelles vécues, ils sont également susceptibles de provoquer de l'émotion.

\footnotetext{
${ }^{13}$ Voir S. Agacinski (Le Monde, 03/02/20 I3) : « Selon le modèle traditionnel, un enfant est rattaché à un parent au moins, généralement la mère qui l'a mis au monde, et si possible à deux, père et mère. $Y$ compris dans l'adoption, la filiation légale reproduit analogiquement le couple procréateur, asymétrique et hétérogène ». Accès : www.lemonde.fr/idees/article/20 I3/02/03/deux-meres-un-pere_| 826278_3232. html\#cvMxhOFwkVyKcGv.99.
} 
Les argumentations peuvent être construites sur une opposition systématique entre spéculation et faits, qui donne la prééminence aux faits. C'est ce que l'on voit par exemple dans l'appel à la réalité pour refuser un débat à coup de slogans et de poncifs. De même, les expériences sensibles, les témoignages faisant appel au vécu, la connaissance du terrain (du milieu), peuvent être utilisés, par exemple pour contredire des principes moraux jugés stériles : « L'argumentation fondée sur l'expérience d'un milieu est au cœur de nombreuses disputes, faisant intervenir la connaissance familière des univers de pratiques ou des dispositifs techniques » (Chateauraynaud, 20 I I :27). Des expressions telles dans la pratique, au quotidien, en réalité... marqueront alors une telle attitude.

Les témoignages, qui sont des rapports subjectifs aux faits, ont une portée émotionnelle incontestable. Dans le débat sur la filiation, l'enfance vécue dans une famille traditionnelle, (mais dysfonctionnelle) et celle dans un environnement familial atypique (et heureux) seront souvent évoquées :

55. «Les enfants conçus par PMA de mes meilleurs amis sont TRÉs heureux et leurs parents aussi. Les \#ManifPourTous sont juste des salauds. »

56. " J'ai toujours vécu avec juste une mère donc vos morales à 2 balles allez vous faire. \# ManifDeLaHonte »

57. « Pour Colette, directrice d'école dans le Xxeme, la famille " un papa-une maman "n'est pas une réalité " sur le terrain " \# AllOu .... »

58. « Mais aussi a venir un témoignage d'une Lesbienne au coeur de la \# ManifPourTous qui crie son mal-être \# ManifDeLaHonte \# Danslactu »

59. «Ton idéologie libérale t'occulte la réalité du monde. La GPA n'est aujourd'hui qu'une entreprise financière. »

II est possible de soutenir (ou de discréditer) une argumentation en mettant en avant (en contestant) l'autorité de celui qui l'énonce. La reconnaissance par les protagonistes de sa crédibilité - fondée à la fois sur la compétence et la sincérité - pèse sur la validité de ses arguments. L'argument d'autorité est l'expression type, souvent caricaturale, du poids de l'énonciateur dans l'argumentation. À l'inverse, l'argument ad hominem cherche à affaiblir la position de l'adversaire en lui opposant ses propres paroles ou actes. La question de la légitimité des protagonistes se décline en réalité selon une multiplicité de cas de figure. Une audience favorable peut être accordée par exemple au témoignage d'une personnalité publique dotée d'un capital de sympathie :

60. « Intervention très réaliste d'Irène Théry sur \# BFMTV à propos de la GPA ! »

61. " "Dans ma famille, on aime la différence, on tolère la différence, on embrasse la différence" \#NoahYannick \#France2tv \#journal \#tolérance »

62. «Comment résumer la crédibilité des participants de la manif pour tous \# ManifDeLaHonte http://»

63. « Je rappelle que le pouvoir qui promet de s'opposer à la \#gpa est le même qui promettait d'inverser la courbe du chômage... \#crédibilité » 
L'appartenance identitaire engage les acteurs dans le choix de postures et d'arguments. On remarquera que le clivage politique est particulièrement présent dans le débat sur la GPA, entrainant avec lui des jugements ciblant des positions supposées être celles de la gauche ou de la droite. Des mots-dièse employés comme termes d'adresse ou commentaires modaux servent à la fois à désigner et à disqualifier l'adversaire : \#GaucheTarée, \#Gauchebarrée, \#gauchecaviar, \#gauchenazie, \#gauchetarée, \#gauchistesdem... De manière plus anecdotique, on retrouvera l'éternel « bobo de gauche » dans les argumentaires des locuteurs de droite. Mais on peut noter que le rejet de cette figure amène des polémiqueurs à le situer automatiquement dans le camp adverse (« Bobo provincial catholique de droite », « LeS RICHES BOBOS GAYS 》, « bobos cathos liberticides », « bobos féministes de Rue de Solférino »...).

Les activités critique et métadiscursive sont intimement liées. Le discours polémique semble immanquablement s'accompagner de représentations axiologiquement orientées d'un discours autre : «Tous les énoncés polémiques [...] se focalisent sur le discours adverse, et sa dénégation » avertit Catherine Kerbrat-Orecchioni ( 1980 : I I-12). Les débatteurs cherchent à s'emparer du discours de l'autre pour mieux le critiquer: dénoncer les manquements de ce discours aux règles de la logique - discours inconsistant, incomplet, délirant, irrationnel, absurde, contradictoire... - et/ ou à celles de la morale - discours pervers, scandaleux, odieux...

Dans le corpus, l'emploi des mots-dièse comme \#contradiction, \#logique ou \#délire sert à dénoncer fortement de tels propos. Plus globalement, une présence récurrente des marques métadiscursives telles justifier, argumentation, raison... confirme une activité argumentative soutenue. Francis Chateauraynaud (201 I : I 17) note que, « dans les phases d'intensification de la dispute, les arguments reçoivent une foule de qualités ». Le corpus FIL en fournit en grand nombre : argument absurde, aucun argument valable, ridicule argument, argument massue, argument pourri, le pire argument, argument bidon, arguments débiles, arguments d'arriérés moyenâgeux... Dans la majorité des cas, le terme argument sert à renvoyer à un élément du discours antagoniste.

64. « Gpa \#pma la rue est un argument massue et ses penseurs des éclaireurs »

65. «Quand l'argument de la \# ManifPourTous c'est que l'enfant a plus de repères pr savoir quel sexe fait la vaisselle, t'as touché le fond »

66. «lls n'ont que le mot "homophobe" à la bouche,ça c'est de l'argumentation \#ManifDeLaHonte »

67. « Je vois pas ou est la logique, ou le truc qu'un enfant serait plus heureux avec un papa et une maman. Je comprends pas. \# ManifDeLaHonte »

68. «N'empêche que les abrutis de la \# manifpourtous sont assez con pour confondre homosexualité, adoption, pédophilie, PMA et GPA. Rien que ça. »

69. «Non par contre y'a contresens quand tu dis vouloir protéger les enfants mais annuler la circulaire naturalisant ceux nés d'une GPA » 


\section{Conclusion}

On rencontre les controverses à toutes les époques et dans tous les domaines. Les hommes sont amenés à confronter leurs idées parce qu'ils diffèrent par les points de vue et les échelles de valeurs qu'ils adoptent à l'égard de leurs environnements et d'eux-mêmes. Même les domaines longtemps perçus comme stables (la santé, la famille, la mort, l'environnement...) deviennent aujourd'hui sources d'enjeux agonistiques. Selon Alain Touraine (2007 : 213), « on pourrait définir les sociétés les plus complexes, comme fragmentées par un grand nombre de conflits qui apparaissent, se développent et sont résolus indépendamment les uns des autres ». Les régimes démocratiques font en sorte que ces divergences puissent se dire, ouvertement, librement et pacifiquement. Les citoyens, eux, cherchent de plus en plus à investir les différents lieux et dispositifs d'expression pour se faire entendre. À la fois instrument et matière, le discours constitue un point d'entrée privilégié pour comprendre ce qui préoccupe une société, ce qui crée et alimente le dissensus.

Nous venons de présenter un ensemble de notions destinées à guider l'interprétation des traces langagières des controverses. L'idée que nous défendons est que le profilage notionnel des productions langagières collectées, souvent foisonnantes, riches et bruitées, permet d'ordonner analytiquement le matériau discursif associé à une controverse. L'enjeu est de pouvoir appréhender des situations polémiques dans leur complexité inhérente, en tenant compte de la diversité des cibles, des attitudes et des points d'achoppement possibles.

Nous avons travaillé sur des productions verbales issues d'un dispositif numérique. Les discours éristiques sur Twitter se distinguent par plusieurs caractéristiques remarquables. La forme courte du tweet conduit les internautes à dire l'essentiel en peu de mots. De ce fait, les verbalisations prennent une forme directe et synthétique. Les opinions défendues et les arguments avancés sont exprimés dans des schémas d'expression simples et stéréotypés. Les débatteurs emploient des mots-dièse pour marquer le thème (ce qui est habituel), mais aussi pour signaler que leur message revêt un caractère polémique (\#contradiction, \#délire, \#duel, \#crédibilité...). Ce matériau langagier révèle donc toute sa pertinence pour nourrir des réflexions autour de la notion même de controverse.

En présence de tels corpus, de nouvelles interrogations émergent. Sommes-nous fondés à penser que les nouvelles formes de communication peuvent induire de nouvelles formes de conflictualité ? Le numérique peut-il sensiblement modifier la nature et la dynamique d'un conflit (influencer son émergence, accélérer la phase de mobilisation, favoriser certains types d'attitudes : agressives, polarisantes, pacifiques...) ? La circulation et le partage facilité des connaissances ainsi que la mise en visibilité des faits de société ont-ils un impact sur les régimes argumentatifs des protagonistes ? Autant de pistes de recherche qui sont à la portée des analystes, à condition de pouvoir intégrer dans des dispositifs d'observation des connaissances et des ressources linguistiques appropriées. 


\section{Références}

Amossy R., Burger M., éds, 201 I, « Polémiques médiatiques et journalistiques. Le discours polémique en questions », Semen, 31 .

Amossy R., 2014, Apologie de la polémique, Paris, Presses universitaires de France.

Angenot M., 1982, La Parole pamphlétaire. Typologie des discours modernes, Paris, Payot.

Angenot M., 2008, Le Dialogue de sourd. Traité de rhétorique antilogique, Paris, Éd. Mille et Une Nuits.

Barats C., éd., 2013, Manuel d'analyse du web, Paris, A. Colin.

Boure R., Bousquet F., 20 I I, « La construction polyphonique des pétitions en ligne. Le cas des appels contre le débat sur l'identité nationale », Questions de communication, 20. Accès : $h$ ttp://questionsdecommunication.revues.org/2/20.

Boyd D., Golder S., Lotan G., 20 I0, «Tweet, Tweet, Retweet: Conversational Aspects of Retweeting on Twitter », in: Proceedings of the $201043^{\text {rd }}$ Hawaii International Conference on System Sciences (HICSS), Washington, Institute of Electrical and Electronics Engineers Computer Society. Accès : http://www.danah.org/papers/TweetTweetRetweet.pdf.

Chateauraynaud F., 201 I , Argumenter dans un champ de forces. Essai de balistique sociologique, Paris, Petra.

Cervulle M., Pailler F., 2014, « \#mariagepourtous: Twitter et la politique affective des hashtags $\gg$, Revue française des sciences de l'information et de la communication, 4. Accès: http://rfsic.revues.org/717.

Dascal M., 1998, «Types of polemics and types of polemical moves », pp. I5-33, in: Cmejrkova S., Hoffmannova J., Mulleriva O., Svetla J., eds, Dialogue Analysis vi, I, Tubingen, M. Niemeyer.

Dascal M., Chang H.-L. eds, 2007, Traditions of Controversy, Amsterdam, J. Benjamins.

Desmons E., Paveau M.-A., éds, 2008, Outrages, insultes, blasphèmes et injures : violences du langage et polices du discours, Paris, Éd. L'Harmattan.

Jackiewicz A., 201 6a, Études sur les discours évaluatifs et d'opinion, Paris, Éd. L'Harmattan.

Jackiewicz A., 20 I 6b, « Outiller l'analyse des controverses. Pourquoi s'intéresser aux discours numériques? », pp. 139-152, in :Wigham C., Ledegen G., dirs, Corpus de communication médiée par les réseaux, Paris, Éd. L'Harmattan.

Jackiewicz A., Vidak M., 20l4, «Étude sur les mots-dièse », pp. 2033-2050, in : Neveu F., Blumenthal P., Hriba L. et al., éds, SHS Web of Conferences, $8,4^{e}$ Congrès mondial de linguistique française, Berlin. Accès : https://www.shs-conferences.org/articles/shsconf/abs/2014/05/ shsconf_cmlfl4_0l 198/shsconf_cmlfl4_0l I98.html.

JulliardV., Cervulle M., 2013, «"Différence des sexes" et controverses médiatiques : du débat sur la parité au "mariage pour tous" | 998-2013 », Le Temps des médias, 21, pp. I6 I - 175.

Kahn A., 20 I 0, Un type bien ne fait pas ça... Morale, éthique et itinéraire personnel, Paris, NiL Éd.

Kerbrat-Orecchioni C., 1980, « La polémique et ses définitions », pp.3-40, in : Gelas N., Kerbrat-Orecchioni C., éds, Le Discours polémique, Lyon, Presses universitaires de Lyon.

Kerbrat-Orecchioni C., 1992, « La négociation interactive des partis pris », pp. 225-235, in : De Mulder W., Schuerewegen F., Tasmowski L., éds, Énonciation et parti pris. Actes du colloque de l'université d'Anvers, 5-6-7 février 1990, Amsterdam, Rodopi. 
Kerbrat-Orecchioni C., 2005, Le Discours en interaction, Paris, A. Colin.

Koren R., 201 I, « De la rationalité et/ou de l'irrationalité des polémiqueurs : certitudes et incertitudes », Semen, 31. Accès : http://semen.revues.org/906।.

Lagorgette D., 2012, « Insulte, injure et diffamation : de la linguistique au code pénal ? », Argumentation et Analyse du discours, 8. Accès : http://aad.revues.org// 312.

Latour B., 2007, « La cartographie des controverses », Technology Review, 0, pp. $82-83$.

Lemieux C., 2007, « À quoi sert l'analyse des controverses? », Mil Neuf Cent. Revue d'histoire intellectuelle, 25 ( I), pp. 191-212.

Longhi J., 20 I3, « Essai de caractérisation du tweet politique », L'Information grammaticale, 136, pp. 25-32.

Maingueneau D. 1983, Sémantique de la polémique, Lausanne, Éd. L'Âge d'homme.

MarwickA. E., Boyd D., 2010 , «I ITweet Honestly, ITweet Passionately:Twitter Users, Context Collaps, and the Imagined Audience », New Media \& Society, I 3 ( I), pp. I | 4- I 33.

Moïse C., Auger N, Fracchiolla B., Schultz-Romain C., éds, 2008, La Violence verbale, 2 tomes, Paris, Éd. L'Harmattan.

Oger C., 20 I2, « La conflictualité en discours : le recours à l'injure dans les arènes publiques 》, Argumentation et Analyse du discours, 8. Accès : http://aad.revues.org/l 297.

Paveau M.-A., 20 I5, « Ce qui s'écrit dans les univers numériques 》, |tinéraires. Littérature, textes, cultures, 20 I4-I. Accès : http://itineraires.revues.org/23 I 3. Dol : 10.4000/itineraires.23 I3.

Plantin C., 1997, « L'argumentation dans l'émotion », Pratiques, 96, pp. 81 - 100.

Plantin C., 2003, « Des polémistes aux polémiqueurs », in : Declercq G., Murat M., Dangel J., éds, La Parole polémique, Paris, H. Champion, pp. 377- 408. Accès : https://halshs. archives-ouvertes.fr/halshs-00425283.

Plantin C., 201 I, Les Bonnes Raisons des émotions. Principes et méthode pour l'étude du discours émotionné, Berne, P. Lang.

Rosier L., éd., 2012, « Insulte, violence verbale, argumentation », Argumentation et Analyse du discours, 8. Accès : https://aad.revues.org// 242.

Rennes J., 2007, «Analyser une controverse. De la science politique à l'étude argumentative », pp. 91-107, in : Bonnafous S., Temmar M., dirs, Analyse de discours et sciences humaines et sociales, Paris, Ophrys.

Reynié D., éd., 2012, Valeurs partagées, Paris, Presses universitaires de France.

StengerT., Coutant A., éds, 201 I, « Ces réseaux numériques dits sociaux », Hermès. La Revue, 59.

Touraine A., 2007, Penser autrement, Paris, Fayard.

Traïni C., éd, 2009, Émotions... Mobilisation!, Paris, Presses de Sciences Po.

Zappavigna M., 20 I I, « Ambient Affiliation: A Linguistic Perspective on Twitter », New Media \& Society, I3 (5). Accès : http://williamwolff.org/wp-content/uploads/20 I3/09/zappavigna-twitterambient-20I I.pdf. 